\title{
A!
}

This is an electronic reprint of the original article.

This reprint may differ from the original in pagination and typographic detail.

Breunig, Daniel; Burset, Pablo; Trauzettel, Björn

\section{Creation of Spin-Triplet Cooper Pairs in the Absence of Magnetic Ordering}

Published in:

Physical Review Letters

DOI:

10.1103/PhysRevLett.120.037701

Published: 19/01/2018

Document Version

Publisher's PDF, also known as Version of record

Please cite the original version:

Breunig, D., Burset, P., \& Trauzettel, B. (2018). Creation of Spin-Triplet Cooper Pairs in the Absence of Magnetic Ordering. Physical Review Letters, 120(3), 1-6. [037701].

https://doi.org/10.1103/PhysRevLett.120.037701

This material is protected by copyright and other intellectual property rights, and duplication or sale of all or part of any of the repository collections is not permitted, except that material may be duplicated by you for your research use or educational purposes in electronic or print form. You must obtain permission for any other use. Electronic or print copies may not be offered, whether for sale or otherwise to anyone who is not an authorised user. 


\title{
Creation of Spin-Triplet Cooper Pairs in the Absence of Magnetic Ordering
}

\author{
Daniel Breunig, ${ }^{1}$ Pablo Burset, ${ }^{2}$ and Björn Trauzettel ${ }^{1}$ \\ ${ }^{1}$ Institute for Theoretical Physics and Astrophysics, University of Würzburg, 97074 Würzburg, Germany \\ ${ }^{2}$ Department of Applied Physics, Aalto University, 00076 Aalto, Finland
}

(Received 11 October 2017; revised manuscript received 20 December 2017; published 19 January 2018)

\begin{abstract}
In superconducting spintronics, it is essential to generate spin-triplet Cooper pairs on demand. Up to now, proposals to do so concentrate on hybrid structures in which a superconductor (SC) is combined with a magnetically ordered material (or an external magnetic field). We, instead, identify a novel way to create and isolate spin-triplet Cooper pairs in the absence of any magnetic ordering. This achievement is only possible because we drive a system with strong spin-orbit interaction - the Dirac surface states of a strong topological insulator (TI)-out of equilibrium. In particular, we consider a bipolar TI-SC-TI junction, where the electrochemical potentials in the outer leads differ in their overall sign. As a result, we find that nonlocal singlet pairing across the junction is completely suppressed for any excitation energy. Hence, this junction acts as a perfect spin-triplet filter across the SC, generating equal-spin Cooper pairs via crossed Andreev reflection.
\end{abstract}

DOI: 10.1103/PhysRevLett.120.037701

Introduction.-In spintronics, it is desirable to achieve spin manipulation in the absence of magnetic fields because electronic switching processes can be done much faster than their magnetic counterparts. The prime example of such a device is the famous Datta-Das transistor based on materials with strong spin-orbit coupling [1]. The situation is similar in superconducting spintronics [2,3]. However, to the best of our knowledge, there is no proposed device that performs in a controlled fashion in the absence of magnetic ordering [4]. Specifically, it would be exciting to create equal-spin Cooper pairs on demand in a device in which spin-orbit coupling is the crucial feature for its application in spintronics. For this vision to come true, the surface states of three-dimensional (3D) topological insulators (TIs) are promising building blocks because they mimic a truly relativistic spin-orbit coupling in a condensed matter setting. We have identified a bipolar device based on two areas of TI surface states connected to each other via a common central superconductor (SC), i.e., a TI-SC-TI junction, that can act as a generator for equal-spin Cooper pairs on demand (cf. Fig. 1). In fact, the working principle of the device is based on crossed Andreev reflection-generated out of equilibrium-which enables a transfer of Cooper pairs (with a finite net spin) from the two TI regions into the SC.

The bipolar TI-SC-TI setup is inspired by a seminal work by Cayssol, where a similar junction has been studied in the context of graphene [5]. In that work, the spin degree of freedom played no role because of the weak spin-orbit coupling in graphene. In TIs, instead, strong spin-orbit coupling in combination with superconducting and/or magnetic ordering gives rise to intriguing physics [6], for instance, the emergence of Majorana bound states
[7-9] or odd-frequency SC pairing [10-14]. The underlying reason is that spin-rotational invariance is broken by spin-orbit coupling and fundamentally different gaps, including a spin-triplet state [15], and can be induced in TI surface states by proximity to an $s$-wave SC and/or a magnetic insulator [16]. From the experimental side, it seems to be more feasible to induce superconducting order into TI surface states than magnetic order, although both tasks have been recently achieved [17-27]. This observation implies that our setup should be directly realizable in the current generation of hybrid structures on TI surfaces.

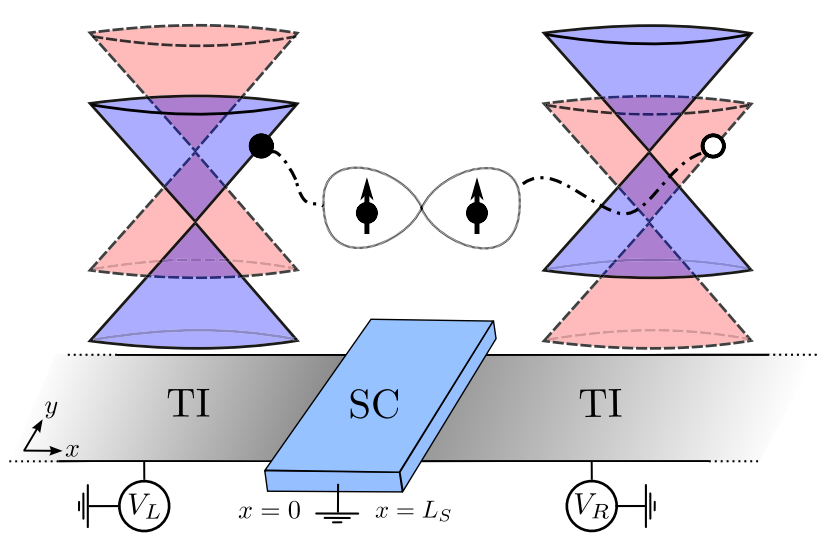

FIG. 1. Schematic of the TI-SC-TI junction. While the voltages applied to the outer leads should be tunable, the SC is assumed to be grounded. The dispersion relations are depicted for illustration: solid (dashed) lines represent electron (hole) Dirac cones. A possible crossed Andreev reflection process-resulting in the injection of equal-spin-triplet Cooper pairs in the SC due to spinmomentum locking-is sketched. 
In this proposal, we assume that an $n$-type region of the Dirac surface states (of a 3D TI) is connected to a $p$-type region via a SC domain, proximity induced by means of a superconducting top electrode; see Fig. 1 for a schematic. The carrier density of each TI domain can be independently tuned by means of local top-gate electrodes [28]. Furthermore, we want the two outer leads to be biased by separate voltage sources [corresponding to biases $V_{L}$ and $V_{R}$ applied to the left $(L)$ and the right $(R)$ TI region, respectively] and the SC to be grounded. In such a bipolar TI-SC-TI junction, Andreev reflection in the same lead and electron tunneling between leads can be efficiently turned off, as previously proposed for graphene [5] and ordinary semiconductors [29]. We show below that, for the case of TIs, spin singlet pairing is strongly suppressed, making this device a perfect spin-triplet filter, where crossed Andreev reflection processes pump equal-spin Cooper pairs into the SC.

Model.-To model the TI-SC-TI device, we introduce the basis $\left[\hat{c}_{\uparrow}(\boldsymbol{k}), \hat{c}_{\downarrow}(\boldsymbol{k}), \hat{c}_{\uparrow}^{\dagger}(-\boldsymbol{k}), \hat{c}_{\downarrow}^{\dagger}(-\boldsymbol{k})\right]^{T}$, where $\hat{c}_{\sigma}^{\dagger}(\boldsymbol{k})$ is the creation operator of an electron with momentum $\boldsymbol{k}$ and spin $\sigma=\uparrow, \downarrow$. In this basis, we can determine the corresponding Bogoliubov-de Gennes (BdG) Hamiltonian

$$
\hat{\mathcal{H}}_{\mathrm{BdG}}=\left(\begin{array}{cc}
H_{0}(\boldsymbol{k}) & i \Delta(x) \boldsymbol{\sigma}_{y} \\
-i \Delta(x) \boldsymbol{\sigma}_{y} & -H_{0}^{*}(-\boldsymbol{k})
\end{array}\right),
$$

where the electron Bloch Hamiltonian reads as

$$
\begin{aligned}
H_{0}(\boldsymbol{k}) & =v_{F}\left(\hat{k}_{x} \boldsymbol{\sigma}_{x}+\hat{k}_{y} \boldsymbol{\sigma}_{y}\right)-\mu(x) \boldsymbol{\sigma}_{0} \\
& \hat{=} v_{F}\left(\hat{k}_{x} \boldsymbol{\sigma}_{x}+k_{y} \boldsymbol{\sigma}_{y}\right)-\mu(x) \boldsymbol{\sigma}_{0} .
\end{aligned}
$$

Here, $v_{F}$ is the Fermi velocity, $\hat{k}_{j}=-i \hbar \partial_{j}$ are the momentum operators in position basis, and $\Delta(x)$ and $\mu(x)$ define spatially dependent SC pairing and electrochemical potentials, respectively. Notice that, in this work, the Pauli matrices $\sigma_{i}$ act on the spin space of the particles, contrary to Ref. [5], where they act on the sublattice space of graphene. We set $v_{F}=\hbar=1$ in what follows. In Eq. (3), we reduce the Bloch Hamiltonian to a quasi-1D operator, where $k_{y}$ is now a parameter defining the angle of incidence. This is possible due to the choice of the coordinate system in Fig. 1 and the perfect alignment of the interfaces (between TI and SC) at $x=0$ and $x=L_{S}$ along the $y$ axis. Experimentally, it is very difficult to resolve the $k_{y}$ dependence. Hence, we will average subsequent characteristics or observables with respect to $k_{y}$. In our TI-SC-TI junction, we assume the electrochemical potentials to be constant in each domain. In particular, we define $\mu(x)=\mu_{L} \Theta(-x)+$ $\mu_{S} \Theta(x) \Theta\left(L_{S}-x\right)+\mu_{R} \Theta\left(x-L_{S}\right)$, with $\Theta(\cdot)$ the Heaviside function. The superconducting pairing is chosen to be finite only underneath the SC, i.e., $\Delta(x)=$ $\Delta_{0} \Theta(x) \Theta\left(L_{S}-x\right)$. This assumption is valid if the Fermi wavelength in the SC region is much smaller than the proximity-induced coherence length.

Setting up the scattering problem for the TI-SC-TI junction (see the Supplemental Material [30]), we obtain the electron and hole wave vectors in the normal leads as $k_{i}^{e / h}=\zeta_{i}^{e / h}\left|\varepsilon \pm \mu_{i}\right| \cos \theta_{i}^{e / h}$, with $i \in\{L, R\}$ and $\varepsilon>0$ the excitation energy. Here, $\zeta_{i}^{e / h}=\operatorname{sgn}\left(\varepsilon \pm \mu_{i}+\left|k_{y}\right|\right)$ defines if the particle stems from the valence or the conduction band and $\theta_{i}^{e / h}=\arcsin \left(k_{y} /\left|\varepsilon \pm \mu_{i}\right|\right)$ is the angle of incidence for electrons or holes. In such a junction, four transport channels exist: (i) normal reflection (NR), (ii) local Andreev reflection (LAR), (iii) electron cotunneling (CO), and (iv) crossed Andreev reflection (CAR).

We obtain a bipolar system by choosing the electrochemical potentials in the TIs to have the same modulus, but different signs, $\mu_{L}=-\mu_{R} \equiv \mu>0$. Because of the nodal dispersion relation, this choice allows us to completely suppress two out of four transport channels: LAR and CO. As illustrated in Fig. 2, this complete suppression is achieved by applying a voltage $V_{L}$ to $L$ such that the corresponding excitation energy coincides with the electrochemical potential, i.e., $e V_{L} \equiv \varepsilon=\mu$. Evidently, by this particular choice, we exactly hit the Dirac point of the hole dispersion in $L$ and the electron dispersion in $R$. Since these states have zero momentum, $\boldsymbol{k}=0$, they do not contribute to transport, completely suppressing LAR and CO.

Superconducting pairing.-Next, we study the symmetry of the superconducting pairing potential that is proximity induced into the TI-SC-TI device. To do so, we analytically compute the retarded Green function using a scattering state approach (see [30]). In the basis defined above, this Green function can be written as $\mathcal{G}^{r}\left(x, x^{\prime}, y, y^{\prime}, k_{y}, \omega\right)=\mathcal{G}^{r}\left(x, x^{\prime}, k_{y}, \omega\right) e^{i k_{y}\left(y-y^{\prime}\right)}$ with

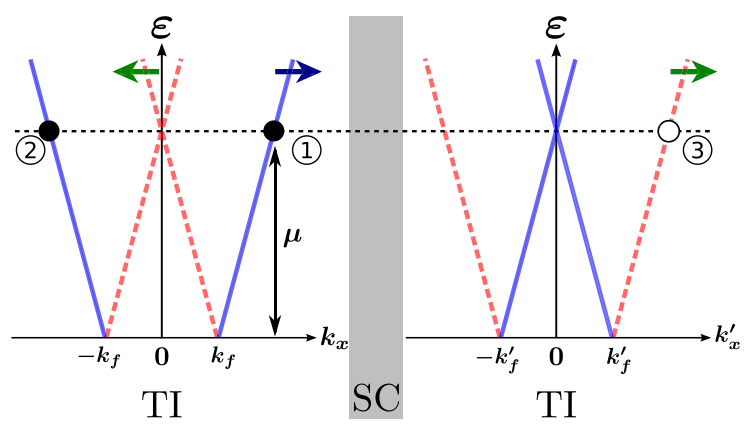

FIG. 2. Electron (solid lines) and hole (dashed lines) dispersion relations in $L$ and $R$, at $k_{y}=0$. Colored arrows on the branches indicate the Cooper pair spin expectation values according to Eq. (16). The SC is depicted by the gray domain. At $\varepsilon=\mu$, the incident electron (1) may be reflected as an electron (2) at the interface (NR) or transmitted as a hole (3) through the SC (CAR). LAR and CO are, instead, not permitted since the states at the Dirac points have zero momentum. 


$$
\begin{gathered}
\mathcal{G}^{r}\left(x, x^{\prime}, k_{y}, \omega\right)=\left(\begin{array}{ll}
\boldsymbol{G}_{e e}^{r} & \boldsymbol{G}_{e h}^{r} \\
\boldsymbol{G}_{h e}^{r} & \boldsymbol{G}_{h h}^{r}
\end{array}\right), \\
\boldsymbol{G}_{n m}^{r}\left(x, x^{\prime}, k_{y}, \omega\right)=\left(\begin{array}{ll}
G_{n m}^{r, \uparrow \uparrow} & G_{n m}^{r, \uparrow \downarrow} \\
G_{n m}^{r, \downarrow \uparrow} & G_{n m}^{r, \downarrow \downarrow}
\end{array}\right) .
\end{gathered}
$$

Dependencies inside the matrices are omitted for ease of notation. Here, $\omega=\varepsilon+i 0^{+}$is the energy shifted infinitesimally into the positive complex plane to impose outgoing boundary conditions [34].

For convenience, we define the anomalous Green function in a rotated basis as

$\mathcal{F}^{r}\left(x, x^{\prime}, k_{y}, \omega\right) \equiv-i \boldsymbol{G}_{e h}^{r} \boldsymbol{\sigma}_{y}=\left(\begin{array}{cc}G_{e h}^{r, \uparrow \downarrow} & -G_{e h}^{r, \uparrow \uparrow} \\ G_{e h}^{r, \downarrow \downarrow} & -G_{e h}^{r, \downarrow \uparrow}\end{array}\right)$,

which we further decompose into singlet or triplet parts

$$
\mathcal{F}^{r}\left(x, x^{\prime}, k_{y}, \omega\right)=\sum_{j \in\{0, x, y, z\}} f_{j}^{r}\left(x, x^{\prime}, k_{y}, \omega\right) \boldsymbol{\sigma}_{j} .
$$

In this equation, $f_{0}^{r}$ is the singlet, and $f_{z}^{r}$ as well as $f_{\uparrow \uparrow / \downarrow \downarrow}^{r} \equiv \mp f_{x}^{r}+i f_{y}^{r}$ are the triplet amplitudes.

Before we discuss specific results on the pairing amplitude for our setup, we state more general arguments based on symmetry. It is straightforward to show that $f_{y}^{r}$ and $f_{z}^{r}$ are odd in $k_{y}$, independent of the choice of $x$ and $x^{\prime}$. Since we later on average all quantities with respect to $k_{y}$, they do not contribute to the (averaged) triplet pairing amplitudes [13]. This implies that $f_{\uparrow \uparrow}^{r}$ and $f_{\downarrow \downarrow}^{r}$ only differ in their sign. The remaining amplitudes $f_{0}^{r}$ and $f_{x}^{r}$ are even functions of $k_{y}$. Hence, they can (in principle) remain finite after averaging.

One of our main results is that this bipolar TI-SC-TI junction acts as a perfect filter for nonlocal triplet pairing in the superconductor because of the helicity of the TI surface states. To substantiate this claim, we first present our results on the nonlocal singlet pairing amplitude. Labeling as $x_{L} \equiv$ $x<0\left(x_{R} \equiv x>L_{S}\right)$ any point in region $L(R)$, we find [35]

$$
f_{0}^{r}\left(x_{L}, x_{R}\right)=\sin \left(\frac{\theta_{L}^{e}-\theta_{R}^{h}}{2}\right) g_{e h}^{\uparrow \downarrow}\left(x_{L}, x_{R}\right),
$$

with

$$
g_{e h}^{\uparrow \downarrow}\left(x_{L}, x_{R}\right)=\frac{\exp \left(i \frac{\theta_{L}^{e}+\theta_{R}^{h}}{2}\right)}{\cos \left(\theta_{L}^{e}\right)} e^{-i\left(k_{L}^{e} x_{L}-k_{R}^{h} x_{R}\right)} d_{1} .
$$

For the nonlocal equal-spin-triplet amplitude, we get

$$
f_{\uparrow \uparrow}^{r}\left(x_{L}, x_{R}\right)=-\frac{i}{2} \frac{e^{i\left(\theta_{L}^{e}+\theta_{R}^{h}\right)}}{\cos \left(\theta_{L}^{e}\right)} e^{i\left(k_{R}^{h} x_{R}-k_{L}^{e} x_{L}\right)} d_{1} .
$$

In these equations, $d_{1}$ denotes the scattering amplitude related to an electron incident from $L$ being transmitted into
$R$ as a hole (see [30]). Similar equations to Eqs. (8)-(10) also apply to the choice $x_{L} \leftrightarrow x_{R}$. In the bipolar regime $\mu_{L}=-\mu_{R} \equiv \mu$, we immediately deduce from the definition of the angles of incidence that $\theta_{L}^{e(h)}=\theta_{R}^{h(e)}$. As a consequence, the nonlocal spin singlet amplitude in Eq. (8) evaluates to zero, making this pairing absent across the junction independently of frequency $\omega$ and mode index $k_{y}$ [36]. In contrast, the nonlocal spin-triplet amplitude in Eq. (10) remains finite (see Fig. 3).

Transport properties.-After the identification of pure triplet pairing across the junction, we want to use this particular property for an application in superconducting spintronics. To achieve this goal, we need to look at nonequilibrium transport properties of the TI-SC-TI junction by means of the (extended) Blonder-TinkhamKlapwijk formalism [37-41]. This allows for the determination of the probability amplitudes of the scattering processes, in general, and the local and nonlocal conductance, in particular. A detailed evaluation of the probability amplitudes (see [30]) confirms the physical picture we elaborated in Fig. 2. For an electron excited in $L$, both the amplitudes for LAR $\left(\equiv R_{1}^{e h}\right)$ and $\mathrm{CO}\left(\equiv T_{1}^{e e}\right)$ vanish at $e V_{L}=\mu$, while those for $\mathrm{NR}\left(\equiv R_{1}^{e e}\right)$ and CAR $\left(\equiv T_{1}^{e h}\right)$ remain finite in the bipolar setup. This property has a striking effect on the nonlinear conductance $\partial I_{L, R} / \partial V_{L}$ at zero temperature $T=0$. Changing only $V_{L}$ and setting $V_{R}=0$, the nonlinear conductance reduces to distinct local and nonlocal parts,

$$
\begin{array}{r}
\frac{\partial I_{L}}{\partial V_{L}}=\frac{2 e^{2}}{h}\left[1-R_{1}^{e e}\left(e V_{L}, k_{y}\right)+R_{1}^{e h}\left(e V_{L}, k_{y}\right)\right], \\
\frac{\partial I_{R}}{\partial V_{L}}=\frac{2 e^{2}}{h}\left[T_{1}^{e e}\left(e V_{L}, k_{y}\right)-T_{1}^{e h}\left(e V_{L}, k_{y}\right)\right] .
\end{array}
$$

The unitarity of the scattering matrix, i.e., $R_{1}^{e e}+R_{1}^{e h}+$ $T_{1}^{e e}+T_{1}^{e h}=1$, yields $T_{1}^{e h}\left(e V_{L}=\mu, k_{y}\right)=1-R_{1}^{e e}\left(e V_{L}=\right.$ $\left.\mu, k_{y}\right)$ and thus

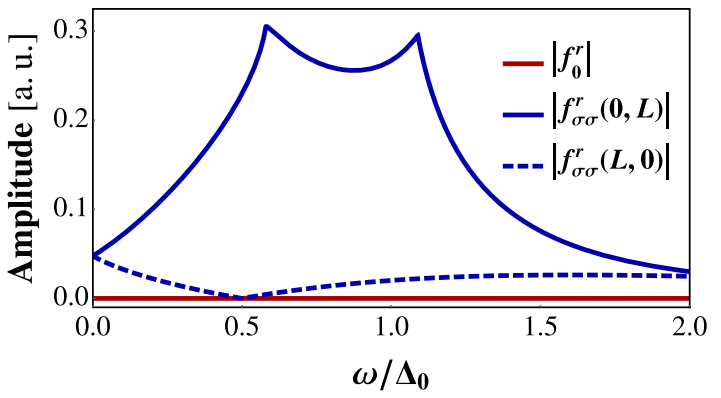

FIG. 3. Averaged moduli of the nonlocal pairing amplitudes from one interface to the other one. For the bipolar setup, nonlocal singlet pairing is completely suppressed, while triplet pairing remains finite. We set $\mu=0.5 \Delta_{0}, \mu_{S}=10 \Delta_{0}$, and $L_{S}=\xi$ $\left(\xi=v_{F} / \Delta_{0}\right)$ with $\sigma \in\{\uparrow, \downarrow\} ; f_{0}^{r}$ illustrates both $f_{0}^{r}(0, L)$ and $f_{0}^{r}(L, 0)$. 


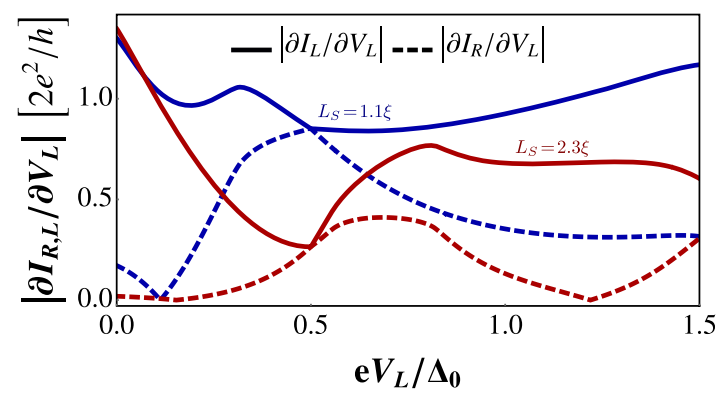

FIG. 4. Averaged moduli of the local and nonlocal conductance for $L_{S}=1.1 \xi$ (blue lines) and $L_{S}=2.3 \xi$ (red lines). At $e V_{L}=\mu$, the curves touch, which is a characteristic feature of the bipolar setup. We set $\mu=0.5 \Delta_{0}$ and $\mu_{S}=10 \Delta_{0}$.

$$
\frac{\partial I_{L}}{\partial V_{L}}\left(e V_{L}=\mu, k_{y}\right)=-\frac{\partial I_{R}}{\partial V_{L}}\left(e V_{L}=\mu, k_{y}\right) .
$$

At this particular point, corresponding to the choice of bias $e V_{L}=\mu$ in our setup, the local and the nonlocal conductance coincide in their absolute value (see Fig. 4). This property is very unusual in superconducting devices because, in general, the local conductance dominates its nonlocal counterpart. Thus, we do not only observe a particular behavior in the nonlocal superconducting pairing, but we are also able to pinpoint a sweet spot at $e V_{L}=\mu$ with striking features in the transport that we can connect to the pumped spin into the SC.

Spin injection.-To do so, we quantify the out of equilibrium spin pumped into the SC via Andreev reflection processes introducing the quantity $\mathcal{S}$, which we denote the nonequilibrium net spin pumped into the SC,

$$
\mathcal{S}=R_{\mathrm{LAR}}\langle\hat{S}\rangle_{l}+T_{\mathrm{CAR}}\langle\hat{S}\rangle_{\mathrm{nl}},
$$

with the local $\langle\cdot\rangle_{l}$ and nonlocal $\langle\cdot\rangle_{\mathrm{nl}}$ contributions

$$
\langle\hat{S}\rangle_{l}=\langle\hat{S}\rangle+\langle\hat{S}\rangle_{\mathrm{LAR}}, \quad\langle\hat{S}\rangle_{\mathrm{nl}}=\langle\hat{S}\rangle+\langle\hat{S}\rangle_{\mathrm{CAR}}
$$

Here, $\hat{S}$ is the Cooper pair spin operator (see [30]) and $\langle\hat{S}\rangle$, $\langle\hat{S}\rangle_{\text {LAR }}$, and $\langle\hat{S}\rangle_{\text {CAR }}$ are the spin expectation values of the incident, the Andreev reflected, and the crossed Andreev reflected particle, respectively. Let us explain why this quantity is a good measure for the spin pumped into the system through triplet Cooper pairs. If we consider, for instance, an electron incident from $L$ moving toward the interface at $x=0$, then there are two processes resulting in the emergence of nonequilibrium Cooper pairs in the SC: (i) LAR with a probability $R_{\mathrm{LAR}}\left(=R_{1}^{e h}\right)$, where the spins of the incident electron and the reflected hole, i.e., $\langle\hat{S}\rangle+\langle\hat{S}\rangle_{\text {LAR }}$, are transferred to the SC; and (ii) CAR with probability $T_{\mathrm{CAR}}\left(=T_{1}^{e h}\right)$, where the spin of the transmitted hole is added to that of the electron, i.e., $\langle\hat{S}\rangle+\langle\hat{S}\rangle_{\mathrm{CAR}}$. Thus, a good estimate of the net spin of the Cooper pairs

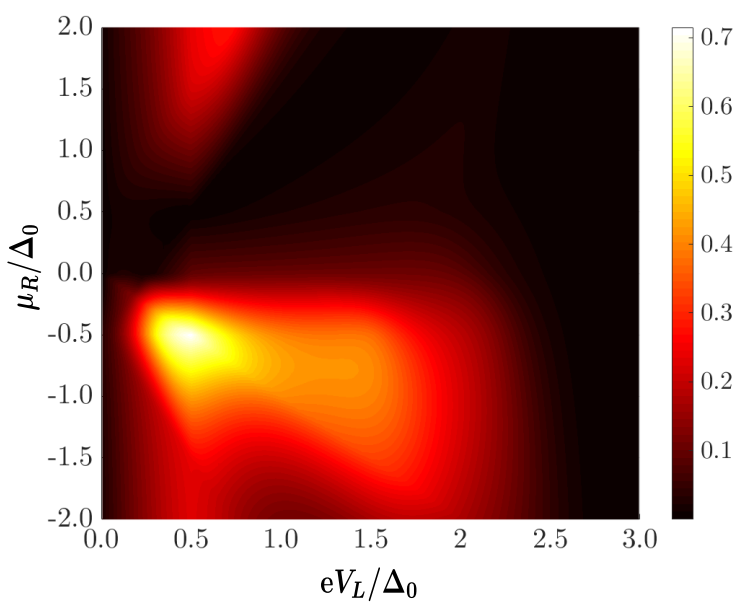

FIG. 5. Nonequilibrium net spin $\mathcal{S}_{x}$ pumped into the $\mathrm{SC}$ as a function of $\mu_{R}$ and $V_{L}$. We set $\mu=0.5 \Delta_{0}, \mu_{S}=10 \Delta_{0}, L_{S}=1.1 \xi$.

pumped into the SC via Andreev reflection is the sum of both contributions $\langle\hat{S}\rangle_{l}$ and $\langle\hat{S}\rangle_{\mathrm{nl}}$, weighted with their respective probability amplitudes. Explicitly, we obtain (under the choice $\hbar=1$ )

$$
\begin{array}{r}
\langle\hat{S}\rangle=\frac{\operatorname{sgn}\left(\varepsilon+\mu_{L}\right)}{2}\left(\zeta_{L}^{e} \cos \theta_{L}^{e}, \sin \theta_{L}^{e}, 0\right)^{T}, \\
\langle\hat{S}\rangle_{\mathrm{LAR}}=\frac{\operatorname{sgn}\left(\varepsilon-\mu_{L}\right)}{2}\left(-\zeta_{L}^{h} \cos \theta_{L}^{h}, \sin \theta_{L}^{h}, 0\right)^{T}, \\
\langle\hat{S}\rangle_{\mathrm{CAR}}=\frac{\operatorname{sgn}\left(\varepsilon-\mu_{R}\right)}{2}\left(\zeta_{R}^{h} \cos \theta_{R}^{h}, \sin \theta_{R}^{h}, 0\right)^{T} .
\end{array}
$$

The norm of these quantities is always $1 / 2$, as expected for fermions, and their $z$-component vanishes. Moreover, their $y$ component is odd under $\theta_{i}^{e / h}$, such that its average with respect to $k_{y}$ will vanish. Thus, only the $x$ component of the net spin (pumped into the SC after averaging over all angles of incidence) remains finite. We can therefore focus on this part of Eqs. (16). Indeed, we find a bound for this component of the net spin

$$
0 \leq\left|\mathcal{S}_{x}\right| \leq R_{\mathrm{LAR}}+T_{\mathrm{CAR}} \leq 1
$$

which corresponds to the maximal angular momentum transferred per scattering event. If we plot this quantity as a function of both $\mu_{R}$ and $e V_{L}$ (see Fig. 5), we find that, in the bipolar setup and for our choice of the SC length $L_{S}$, we pump the largest amount of nonequilibrium net spin into the SC by tuning the bias in the vicinity of the sweet spot $e V_{L}=\mu$.

Summary.-The breaking of spin-rotational invariance in materials with strong spin-orbit locking reveals striking physics. We found that, by connecting a $p$-type to an $n$-type TI via a SC domain, this bipolar TI-SC-TI junction acts as an effective nonlocal spin-triplet filter, where nonlocal 
singlet pairing is completely suppressed. Because of the helicity of the TI states, the nonequilibrium triplet Cooper pairs pumped into the SC carry a finite net spin. We thus propose this setup as an all-electric nanostructure for applications in spintronics. The effect of the spin pumping is strongest in the vicinity of the sweet spot $e V_{L}=\mu_{L}=$ $-\mu_{R}$, where we estimate a net spin of, depending on the bias range, approximately $\mathcal{S}_{x} \approx 0.6-1.2 \hbar$ per unit volume (see [30]) for a SC length of $L_{S}=1.1 \xi$. This sweet spot is experimentally easy to detect, since the local and the nonlocal conductivities for a bias applied to the left TI coincide in their moduli. Interestingly, equal-spin Cooper pairs pumped into a superconductor out of equilibrium could lead to long-range spin accumulation [42,43]. Connecting a second superconductor to the central region of the bipolar junction, the spin accumulation could be measured through a local superconducting quantum interference device or Hall probe [44-46].

We thank Y. Asano, C. W. J. Beenakker, F. S. Bergeret, F. Crepin, F. Dominguez, J. Linder, J. Pekola, B. Scharf, S. Zhang, and N. Traverso Ziani for interesting discussions. Financial support by the DFG (SPP1666 and SFB1170 "ToCoTronics"), the Helmholtz Foundation (VITI), and the ENB Graduate School on "Topological Insulators" is gratefully acknowledged. P. B. acknowledges funding from the European Union's Horizon 2020 research and innovation program under the Marie Skłodowska-Curie Grant No. 743884.

[1] S. Datta and B. Das, Appl. Phys. Lett. 56, 665 (1990).

[2] M. Eschrig, Phys. Today 64, No. 1, 43 (2011).

[3] J. Linder and J. W. A. Robinson, Nat. Phys. 11, 307 (2015).

[4] Many proposals for superconducting spin valves require the use of magnetic elements. See, for example, S. Takahashi, H. Imamura, and S. Maekawa, Phys. Rev. Lett. 82, 3911 (1999); Y. V. Fominov, A. A. Golubov, and M. Y. Kupriyanov, JETP Lett. 77, 510 (2003); K. Halterman, P. H. Barsic, and O. T. Valls, Phys. Rev. Lett. 99, 127002 (2007); K. Halterman, O. T. Valls, and M. Alidoust, Phys. Rev. Lett. 111, 046602 (2013); Y. V. Fominov, A. A. Golubov, T. Y. Karminskaya, M. Y. Kupriyanov, R. G. Deminov, and L. R. Tagirov, JETP Lett. 91, 308 (2010); P. V. Leksin, N. N. Garif'yanov, I. A. Garifullin, Y. V. Fominov, J. Schumann, Y. Krupskaya, V. Kataev, O. G. Schmidt, and B. Büchner, Phys. Rev. Lett. 109, 057005 (2012); L. Y. Zhu, Y. Liu, F. S. Bergeret, J. E. Pearson, S. G. E. te Velthuis, S. D. Bader, and J. S. Jiang, Phys. Rev. Lett. 110, 177001 (2013); A. Singh, S. Voltan, K. Lahabi, and J. Aarts, Phys. Rev. X 5, 021019 (2015); N. Banerjee, C. B. Smiet, R. G. J. Smits, A. Ozaeta, F. S. Bergeret, M. G. Blamire, and J. W. A. Robinson, Nat. Commun. 5, 3048 (2014).

[5] J. Cayssol, Phys. Rev. Lett. 100, 147001 (2008).

[6] J. Linder, Y. Tanaka, T. Yokoyama, A. Sudbø, and N. Nagaosa, Phys. Rev. Lett. 104, 067001 (2010).

[7] L. Fu and C. L. Kane, Phys. Rev. Lett. 100, 096407 (2008).
[8] J. Nilsson, A. R. Akhmerov, and C. W. J. Beenakker, Phys. Rev. Lett. 101, 120403 (2008).

[9] Y. Tanaka, T. Yokoyama, and N. Nagaosa, Phys. Rev. Lett. 103, 107002 (2009).

[10] T. Yokoyama, Phys. Rev. B 86, 075410 (2012).

[11] A. M. Black-Schaffer and A. V. Balatsky, Phys. Rev. B 86, 144506 (2012).

[12] F. Crépin, P. Burset, and B. Trauzettel, Phys. Rev. B 92 , 100507 (2015).

[13] P. Burset, B. Lu, G. Tkachov, Y. Tanaka, E. M. Hankiewicz, and B. Trauzettel, Phys. Rev. B 92, 205424 (2015).

[14] J. Cayao and A. M. Black-Schaffer, Phys. Rev. B 96, 155426 (2017).

[15] G. Tkachov and E. M. Hankiewicz, Phys. Status Solidi (b) 250, 215 (2013).

[16] Triplet superconductivity naturally arises in materials or proximitized structures with broken spin-rotation symmetry: P. A. Frigeri, D. F. Agterberg, A. Koga, and M. Sigrist, Phys. Rev. Lett. 92, 097001 (2004); 93, 099903(E) (2004).A. G. Mal'shukov and C. S. Chu, Phys. Rev. B 78, 104503 (2008); P. Burset, F. Keidel, Y. Tanaka, N. Nagaosa, and B. Trauzettel, Phys. Rev. B 90, 085438 (2014); C. R. Reeg and D. L. Maslov, Phys. Rev. B 92, 134512 (2015); F. Konschelle, I. V. Tokatly, and F. S. Bergeret, Phys. Rev. B 92, 125443 (2015); F. S. Bergeret and I. V. Tokatly, Phys. Rev. B 94, 180502 (2016); I. V. Bobkova and A. M. Bobkov, Phys. Rev. B 95, 184518 (2017).

[17] M.-X. Wang, C. Liu, J.-P. Xu, F. Yang, L. Miao, M.-Y. Yao, C. L. Gao, C. Shen, X. Ma, X. Chen, Z.-A. Xu, Y. Liu, S.-C. Zhang, D. Qian, J.-F. Jia, and Q.-K. Xue, Science 336, 52 (2012).

[18] P. Zareapour, A. Hayat, S. Y. F. Zhao, M. Kreshchuk, A. Jain, D. C. Kwok, N. Lee, S.-W. Cheong, Z. Xu, A. Yang, G. Gu, S. Jia, R. J. Cava, and K. S. Burch, Nat. Commun. 3, 1056 (2012).

[19] B. Sacépé, J. B. Oostinga, J. Li, A. Ubaldini, N. J. Couto, E. Giannini, and A. F. Morpurgo, Nat. Commun. 2, 575 (2012).

[20] L. Maier, J. B. Oostinga, D. Knott, C. Brüne, P. Virtanen, G. Tkachov, E. M. Hankiewicz, C. Gould, H. Buhmann, and L. W. Molenkamp, Phys. Rev. Lett. 109, 186806 (2012).

[21] M. Veldhorst, M. Snelder, M. Hoek, T. Gang, V. K. Guduru, X. L. Wang, U. Zeitler, W. G. van der Wiel, A. A. Golubov, H. Hilgenkamp, and A. Brinkman, Nat. Mater. 11, 417 (2012).

[22] J. R. Williams, A. J. Bestwick, P. Gallagher, S. S. Hong, Y. Cui, A.S. Bleich, J. G. Analytis, I. R. Fisher, and D. Goldhaber-Gordon, Phys. Rev. Lett. 109, 056803 (2012).

[23] S. Cho, B. Dellabetta, A. Yang, J. Schneeloch, Z. Xu, T. Valla, G. Gu, M. J. Gilbert, and N. Mason, Nat. Commun. 4, 1689 (2013).

[24] J. B. Oostinga, L. Maier, P. Schüffelgen, D. Knott, C. Ames, C. Brüne, G. Tkachov, H. Buhmann, and L. W. Molenkamp, Phys. Rev. X 3, 021007 (2013).

[25] I. Sochnikov, L. Maier, C. A. Watson, J. R. Kirtley, C. Gould, G. Tkachov, E. M. Hankiewicz, C. Brüne, H. Buhmann, L. W. Molenkamp, and K. A. Moler, Phys. Rev. Lett. 114, 066801 (2015).

[26] J. Wiedenmann, E. Bocquillon, R. S. Deacon, S. Hartinger, O. Herrmann, T. M. Klapwijk, L. Maier, C. Ames, C. Brüne, 
C. Gould, A. Oiwa, K. Ishibashi, S. Tarucha, H. Buhmann, and L. W. Molenkamp, Nat. Commun. 7, 10303 (2016).

[27] C. Lee, F. Katmis, P. Jarillo-Herrero, J. S. Moodera, and N. Gedik, Nat. Commun. 7, 12014 (2016).

[28] J. Wiedenmann, E. Liebhaber, J. Kübert, E. Bocquillon, P. Burset, C. Ames, H. Buhmann, T. M. Klapwijk, and L. W. Molenkamp, Phys. Rev. B 96, 165302 (2017).

[29] M. Veldhorst and A. Brinkman, Phys. Rev. Lett. 105, 107002 (2010).

[30] See Supplemental Material at http://link.aps.org/ supplemental/10.1103/PhysRevLett.120.037701, which includes Refs. [31-33], where we give explicit definitions, derivations, and calculations on the scattering problem and the transport properties, on the Green function, and on the spin characteristics.

[31] W. L. McMillan, Phys. Rev. 175, 559 (1968).

[32] S. Kashiwaya, Y. Tanaka, M. Koyanagi, and K. Kajimura, Phys. Rev. B 53, 2667 (1996).

[33] S. Kashiwaya and Y. Tanaka, Rep. Prog. Phys. 63, 1641 (2000).

[34] S. Datta, Electronic Transport in Mesoscopic Systems (Cambridge University Press, Cambridge, England, 2007).

[35] The expressions in Eqs. (8)-(10) are derived under the condition $0<-\mu_{R}<\mu_{L}<\varepsilon$. They capture the spin amplitudes correctly as we approach the bipolar regime.
[36] The local spin singlet, where the two electrons forming the Cooper pair are taken from the same region, is proportional to the LAR amplitude. It is thus also suppressed at the bipolar junction.

[37] G. E. Blonder, M. Tinkham, and T. M. Klapwijk, Phys. Rev. B 25, 4515 (1982).

[38] M. P. Anantram and S. Datta, Phys. Rev. B 53, 16390 (1996).

[39] C. J. Lambert and R. Raimondi, J. Phys. Condens. Matter 10, 901 (1998).

[40] G. Falci, D. Feinberg, and F. W. J. Hekking, Europhys. Lett. 54, 255 (2001).

[41] J. Linder and A. Sudbø, Phys. Rev. B 77, 064507 (2008).

[42] M. Silaev, P. Virtanen, F. S. Bergeret, and T. T. Heikkilä, Phys. Rev. Lett. 114, 167002 (2015).

[43] F. S. Bergeret, M. Silaev, P. Virtanen, and T. T. Heikkila, arXiv:1706.08245).

[44] P. G. Björnsson, Y. Maeno, M. E. Huber, and K. A. Moler, Phys. Rev. B 72, 012504 (2005).

[45] J. R. Kirtley, C. Kallin, C. W. Hicks, E.-A. Kim, Y. Liu, K. A. Moler, Y. Maeno, and K. D. Nelson, Phys. Rev. B 76, 014526 (2007).

[46] K. Sengupta and V. M. Yakovenko, Phys. Rev. Lett. 101, 187003 (2008). 